\title{
DEEP-South: Preliminary Photometric Results from the KMTNet-CTIO
}

\author{
Myung-Jin Kim*, Hong-Kyu Moon, Young-Jun Choi, \\ Hong-Suh Yim, Young-Ho Bae, Dong-Goo Roh, \\ Jin Tae Park, Bora Moon and the DEEP-South Team
}

Korea Astronomy and Space Science Institute, 776, Daedeokdae-ro, Yuseong-gu, Daejeon, Republic of Korea * email: skarma@kasi.re.kr

\begin{abstract}
Korea Astronomy and Space Science Institute (KASI) successfully completed the development of Korea Microlensing Telescope Network (KMTNet, Park et al. 2012) in mid-2015, following which it conducted test runs for several months. 'DEep Ecliptic Patrol of the Southern sky' (DEEP-South, Moon et al. 2015), which will be used for asteroid and comet studies, will not only characterize targeted asteroids, carrying out blind surveys toward the sweet spots, but will also mine the data of such bodies using the KMTNet archive. We report preliminary lightcurves of four Potentially Hazardous Asteroids (PHAs) from test runs at KMTNet-CTIO in the February - May 2015 period.
\end{abstract}

Keywords. minor planets, asteroids

\section{Overview}

KMTNet consists of three identical $1.6 \mathrm{~m}$ prime focus telescopes, each equipped with $18 \mathrm{k} \times 18 \mathrm{k}$ mosaic CCDs, resulting in $2 \times 2$ degrees of field of view. The three stations (CTIO in Chile, SAAO in South Africa and SSO in Australia) are longitudinally well separated, and thus will benefit from 24-h continuous monitoring of the southern sky. We awarded 45 full nights every year at each site for five years (2015 - 2019) during the "non-bulge season". We carried out test runs at KMTNet-CTIO in the fall 2015 season, and plan to carry out normal operation at three KMTNet stations from early October 2015 .

\section{Test run targets}

The primary scientific objective of DEEP-South is physical characterization of km-class PHAs at opposition. We conducted targeted observations to achieve this goal during our test runs in the February - May period in 2015. The five different observation modes for DEEP-South are: Opposition Census (OC), Sweet spot survey (S1), Ecliptic survey (S2), NEOWISE follow-up (NW), and Target of Opportunity observation (TO) (Moon et al. 2015). Target information and observed dates are shown in Table 1.

\section{Lightcurves}

We present four individual PHAs' lightcurves obtained from the test runs in Figure 1. All figures were drawn as composite lightcurve folded with their synodic rotational periods. The horizontal axis of each lightcurve represents rotational phase, and the vertical axis represents differential magnitude between instrumental magnitude of an asteroid 
Table 1. Target asteroids obtained from test run at KMTNet-CTIO in February - May 2015 period

\begin{tabular}{|c|c|c|c|c|}
\hline Class & Objects & & $\mathbf{P}_{\text {rot }}(\mathbf{h r})$ & Observing dates (UT) \\
\hline \multirow[t]{3}{*}{ PHA } & $\begin{array}{l}4015 \text { Wilson- } \\
\text { Harrington }\end{array}$ & 15.99 & 3.5736 & 24 Feb 2015 \\
\hline & $5189(1990$ UQ $)$ & 17.9 & 6.65 & 26 Feb, 08, 10 Mar, 11, 13 Apr 2015 \\
\hline & $\begin{array}{l}\text { 12923 Zephyr } \\
(1999 \text { GK4) }\end{array}$ & 15.8 & 3.891 & 26 Feb, 08, 12 Mar, 07, 09 Apr 2015 \\
\hline | & $52760(1998$ ML14) & 17.5 & 14.28 & 02 Mar 2015 \\
\hline \multirow[t]{2}{*}{ | } & 53319 (1999 JM8) & 15.2 & 136. & 14 Feb 2015 \\
\hline & 53426 (1999 SL5) & 17.1 & 2.93 & $\begin{array}{c}12 \mathrm{Feb}, 04,14,16 \mathrm{Mar}, 22,24 \mathrm{Apr} \\
04,06 \text { May } 2015\end{array}$ \\
\hline | & 89830 (2002 CE) & 14.9 & 2.6149 & $11,13,22,24$ Apr 2015 \\
\hline | & $99248(2001$ KY66) & 16.4 & 19.7 & 12, 14, 26 Feb, 02 Mar 2015 \\
\hline 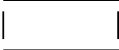 & $136617(1994 \mathrm{CC})$ & 17.7 & 2.3886 & 24 Feb 2015 \\
\hline | & $186844(2004$ GA1) & 17.4 & unknown & 14 Feb 2015 \\
\hline | & $212546(2006$ SV19) & 17.7 & unknown & 28 Feb, 06, 10 Mar 2015 \\
\hline | & $242450(2004$ QY2) & 14.7 & unknown & 11,13 Apr 2015 \\
\hline | & | $311044(2004 \mathrm{BB} 103)$ & 17.1 & unknown & 14 Feb 2015 \\
\hline & | $363790(2005 \mathrm{JE} 46)$ & 17.7 & unknown & 02 Mar 2015 \\
\hline & 385186 (1994 AW1) & 17.6 & 2.5193 & $14 \mathrm{Feb}, 12,16$ Mar 2015 \\
\hline | & $414287(2008$ OB9) & 17.7 & 42.5 & 28 Feb, 06 Mar, 11, 13 Apr 2015 \\
\hline | & $430440(2000 \mathrm{OH})$ & 17.4 & unknown & 12 Feb, 04, 14 Mar, 07, 09 Apr 2015 \\
\hline | & (2007 CA19) & 17.4 & unknown & 14 Feb 2015 \\
\hline | NHATS | & 293726 (2007 RQ17) & 22.6 & | unknown & 24, 26, 30 May, 01 Jun 2015 \\
\hline \multirow[t]{2}{*}{ | } & $416186(2002$ TD 60$)$ & 19.3 & 2.8513 & 10, 14, May 2015 \\
\hline & 436724 (2011 UW 158) & 19.5 & 0.61 & $\begin{array}{c}26,28,30 \mathrm{Apr}, \\
02,08,12,16,18,20,22,26,28 \text { May } 2015\end{array}$ \\
\hline \multirow[t]{2}{*}{ Comet } & | 119P/Parker-Hartley & - & - & 06 Mar 2015 \\
\hline & $269 \mathrm{P} /$ Jedicke & - & - & 06 Mar 2015 \\
\hline | & |2014W11 (PANSTARRS) & - & - & 06 Mar 2015 \\
\hline $\mid$ & | 2015 A1 (PANSTARRS) & - & - & 06 Mar 2015 \\
\hline
\end{tabular}

Notes:

Rotational periods $\mathrm{P}_{\text {rot }}$ (hr) adopted from LCDB (Warner et al. 2009) (Rev. 2015-May). For three objects (1990 UQ, 1999 SL5, and 2011 UW 158) marked with boldface, $\mathrm{P}_{\text {rot }}$ were obtained for the first time.

and an average magnitude of comparison stars. This is the first time that we have derived the rotational periods of asteroids 5189 (1990 UQ) and 53426 (1999 SL5) from our observations. 385186 (1994 AW1) is one of the well-known binary NEAs, with a primary rotational period of $2.5193 \mathrm{hr}$ (Pravec \& Hahn 1997).

Asteroid 436724 (2011 UW158) is listed on the Jet Propulsion Laboratory (JPL)'s NHATS (Near-Earth Object Human Space Flight Accessible Target Study) table; its size has been estimated to be $\sim 300 \times 600 \mathrm{~m}(\mathrm{H}=19.5)$ with the radio telescope at Arecibot. Based on our time series photometry at KMTNet-CTIO in April 2015, we

$\dagger$ Solar System Studies at Arecibo Observatory, http://www.naic.edu/ pradar/news.php\#2015 

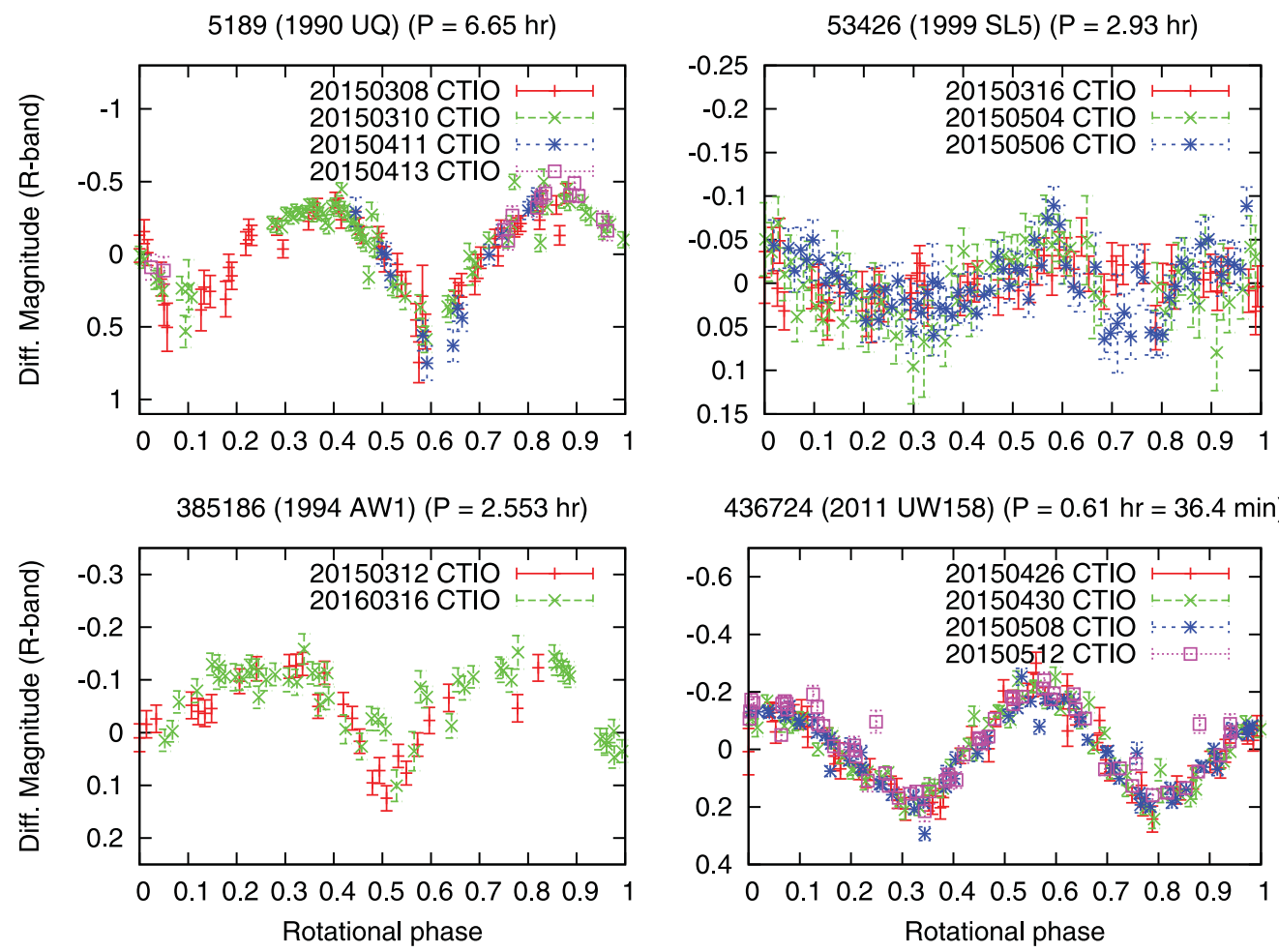

Figure 1. Composite lightcurves of 5189 (1990 UQ), 53426 (1999 SL5), 385186 (1994 AW1) and 436724 (2011 UW158) folded with their rotational periods.

found a synodic rotational period of $0.61 \mathrm{hr}(\sim 36.4 \mathrm{~min})$ and amplitude of $0.5 \mathrm{mag}$, for the first time. The 3D-shape and rotational period of this elongated rapid rotator was confirmed by Goldstone-GBT radar observationsł in July 2015.

\section{Implications}

Round-the-clock observations with the KMTNet telescopes are considered to be optimized for spin characterization of asteroids, including tumbling and slowly-rotating bodies, and are expected to facilitate the debiasing of previously reported lightcurve observations. For the sake of efficiency, we implemented an automated observation scheduler, SMART (Scheduler for Measuring Asteroids RoTation, Kim 2014), which is designed to conduct follow-up observations on time with another, to effectively fill in the period gaps. It automatically updates ephemerides, checks priorities, prepares target lists, and sends a suite of scripts to site operators. We also revised the mover detection software, MODP (Moving Object Detection Program, Bae et al. 2005) and ASAP (Asteroid Spin Analysis Package, Kim 2014) that aids to find a set of comparison stars in each CCD image, to derive spin parameters and reconstruct lightcurve simultaneously in a semi-automatic manner (Yim et al. 2015). In addition to spin characterization of a large number of asteroids and comets, either in near Earth space or in the main-belt, the KMTNet software subsystem with near-real time data reduction and analysis capabilities is expected to serendipitously discover and characterize outer Solar System objects at the same time.

$\ddagger$ Goldstone Radar Observations Planning, http://echo.jpl.nasa.gov/asteroids/2011UW158/ 2011UW158_planning.html 


\section{References}

Bae, Y.-H., Byun, Y.-I., Kang, Y.-W., Park, S.-Y., Oh, S.-H., Yu, S.-Y., Han, W., Yim, H.-S., \& Moon, H.-K. 2015, JASS, 22, 393

Kim, M.-J. 2014, PhD thesis, Yonsei Univ.

Moon, H.-K., Kim, M.-J., Yim, H.-S., Choi, Y.-J., Bae Y.-H., Roh, D.-G., Park, J., \& Moon, B., the DEEP-South Team 2015, Asteroids: New Observations, New Models Proc. IAU Symposium No. 318, this volume

Park, B.-G., Kim, S.-L., Lee, J. W., Lee, B.-C., Lee, C.-U., Han, C., Kim, M., Moon, D.-S., Moon, H.-K., Rey, S.-C., Sung, E.-C., \& Sung, H. 2012, Ground-based and Airborne Telescopes IV Proc. of the SPIE, 8444, 47

Pravec, P. \& Hahn, G. 1997, Icarus, 127, 431

Warner, B. D., Harris, A. W., \& Pravec, P. 2009, Icarus, 202, 134

Yim, H.-S., Kim, M.-J., Bae, Y.-H., Moon, H.-K., Choi, Y.-J., Roh, D.-G., Park, J. T., \& Moon, B., the DEEP-South Team 2015, Asteroids: New Observations, New Models Proc. IAU Symposium No. 318, this volume 